\title{
DBT-galactography: a promising tool for improving the diagnostic workup of nipple discharge
}

\author{
Marco Moschetta ${ }^{1 *}$, Vincenzo De Ruvo ${ }^{1}$, Angelica Drago ${ }^{2}$, Nicoletta Troiano², Simona Paolicelli ${ }^{2}$, Giuseppe Rubini ${ }^{2}$, \\ Amato Antonio Stabile lanora ${ }^{2}$ and Michele Telegrafo ${ }^{1}$
}

\begin{abstract}
Background: Our aim was to compare the diagnostic performance of digital breast tomosynthesis (DBT)galactography with that of full-field digital (FFD)-galactography for detecting intraductal breast lesions using an intra-individual design.

Methods: Forty-nine consecutive patients with spontaneous, unilateral, single-pore nipple discharge and inconclusive FFD mammography and ultrasonography underwent galactography with a "COMBO" technique combining FFD- and DBT-galactography acquisitions. Examinations were independently analysed by two breast radiologists with 10-year experience. Sensitivity, specificity, and accuracy for both FFD- and DBT-galactography were calculated having histological examinations of surgical specimens as a reference standard. Data were presented as percentages with their $95 \%$ confidence intervals (Cl). McNemar test was used. Interobserver agreement was assessed by using Cohen $\mathrm{k}$ test for both techniques.

Results: Sensitivity was 41/43 (95\%, 95\% Cl 84.2-99.4) for DBT-galactography and 33/43 (77\%, 95\% Cl 61.4-88.2) for FFD-galactography $(p=0.008)$, specificity $6 / 6(100 \%, 95 \%$ Cl $54.1-100.0)$ for both imaging tools, accuracy $47 / 49$ (96\%, 95\% Cl 86.0-99.5) and 39/49 (80\%, 95\% Cl 65.7-89.8) ( $p=0.038)$, respectively. The inter-observer agreement was 0.86 for DBT-galactography and 0.78 for FFD-galactography. The AGD resulted to $1.94 \pm 0.64$ for the combined technique.

Conclusion: DBT-galactography showed a significantly higher sensitivity and accuracy than FFD-galactography for the identification of the intraductal findings, improving the possibility of a reliable diagnosis in patients with pathologic nipple-discharge.
\end{abstract}

Keywords: Breast, Digital breast tomosynthesis, Galactography, Mammography, Nipple discharge

\section{Key points}

- Digital breast tomosynthesis (DBT)-galactography and full-field digital (FFD)-galactography were compared in 49 patients with pathological nipple discharge and negative mammography and ultrasonography.

\footnotetext{
* Correspondence: marco.moschetta@gmail.com

'DETO-Department of Emergency and Organ Transplantation-Breast Care Unit, Aldo Moro University of Bari Medical School, Piazza Giulio Cesare 11, 70124 Bari, Italy

Full list of author information is available at the end of the article
}

- DBT-galactography showed a significantly higher sensitivity (95\%) than FFD-galactography (77\%) for the identification of intraductal pathologic findings, without trade-off in terms of specificity (100\% for both techniques).

- DBT-galactography may become an important diagnostic step in managing pathological nipple discharge.

\section{Background}

Nipple discharge represents the third most common breast complaint after pain and lump, with a reported

\section{Springer Open}

(c) The Author(s). 2020 Open Access This article is licensed under a Creative Commons Attribution 4.0 International License, which permits use, sharing, adaptation, distribution and reproduction in any medium or format, as long as you give appropriate credit to the original author(s) and the source, provide a link to the Creative Commons licence, and indicate if changes were made. The images or other third party material in this article are included in the article's Creative Commons licence, unless indicated otherwise in a credit line to the material. If material is not included in the article's Creative Commons licence and your intended use is not permitted by statutory regulation or exceeds the permitted use, you will need to obtain permission directly from the copyright holder. To view a copy of this licence, visit http://creativecommons.org/licenses/by/4.0/. 
prevalence of 7-10\%. Most nipple discharges are benign or not associated with an underlying breast disease [1-3]. On the other side, the most common causes of pathologic nipple discharge consist in benign lesions, such as intraductal papilloma and papillomatosis occurring in $48 \%$ of cases, ductal ectasia in 15-20\% or malignant lesions, such as papillary carcinoma, ductal carcinoma in situ (DCIS) and invasive ductal carcinoma with a high variable rate ranging from 1 to $45 \%$ of patients [3-5].

The diagnostic workup of pathologic nipple discharge still remains a controversial topic, and no general consensus exists in the medical literature. Mammography and ultrasonography are the first-line imaging techniques for evaluating nipple discharge, followed by cytological analysis of the secretion material [6]. Mammographic findings have been reported to be positive in 50 to $90 \%$ of patients with malignancy and in less than $50 \%$ of patients with a benign papilloma. In fact, mammography has some limitations in demonstrating small lesions, in case of lack of microcalcifications and if lesions are completely intraductal [7]. Ultrasonography of papillary lesions typically shows a solid, oval, intra-ductal mass, often associated with duct dilatation. A cystic component is also commonly seen $[1,8,9]$.

Unfortunately, these methods present some limitations in evaluating intraductal lesions; therefore, negative examinations or benign findings cannot exclude an underlying malignant disease $[8,9]$. Recently, the role of unenhanced and contrast-enhanced magnetic resonance imaging (MRI) of the breast for identifying and characterising lesions associated with nipple discharge has been emphasised due to its diagnostic performance. A meta-analysis including a total of 921 patients [10] showed a pooled sensitivity for any abnormality significantly higher for MRI (92\%) than for galactography (69\%) associated with a significantly higher pooled specificity (76\% versus $39 \%$, respectively); the pooled sensitivity and specificity for cancer detection were obtained for MRI only and were $92 \%$ and $97 \%$, respectively. However, the routine use of MRI is still not recommended in routine clinical practice of nipple discharge; it can be useful in selected cases for evaluating inconclusive mammography and US [6].

For a long time, galactography (also named "ductography" or "ductogalactography"), performed after retrograde filling of the lactiferous duct with iodinated contrast material in case of spontaneous, unilateral, single-pore nipple discharge, has been considered as the standard-of-care technique, being effective in identifying and locating breast intraductal lesions [11-14]. However, the role of galactography has remained unclear with some authors recommending this tool for evaluating nipple discharge while others considering it no longer acceptable in the modern era of breast imaging [6]. The specificity and sensitivity of this diagnostic examination is widely influenced by the overlapping of different structures on the contrast-enhanced two-dimensional mammograms.
On the other hand, digital breast tomosynthesis (DBT) has been demonstrated to have higher accuracy compared with digital mammography being able to provide sectional images from different projection angles, avoiding the lesion masking effect due to the tissue superposition [15]. DBT-galactography, i.e., the usage of DBT instead of standard full-field digital (FFD) mammography after retrograde filling of the lactiferous duct with iodinated contrast material, has been investigated only in a recent study evaluating only five patients with promising results [16].

The aim of our study was to evaluate the diagnostic value of DBT-galactography for detecting intraductal breast lesion in the clinical setting of pathological nipple discharge and to compare its performance with that of FFD-galactography, having the histological findings as a reference standard.

\section{Methods \\ Population}

This retrospective study was approved by the University of Bari Medical School-D.E.T.O.-Department of Emergency and Organ Transplantation on January 2019. Between January 2018 and February 2019, all consecutive patients with spontaneous, unilateral, single-pore nipple discharge and negative breast ultrasonography were enrolled and underwent both FFD and DBT-galactography. Written informed consent was obtained from all patients according to the Declaration of Helsinki principles.

Patients with nipple retraction or a history of a prior nipple surgery were excluded from the study. Also patients with US findings suggesting intraductal lesions and eligible for US-guided breast biopsy $(n=21)$ were excluded from the study and did not undergo galactography.

In all cases, cytological analysis of nipple discharge material was performed. The breast was gently massaged in the direction of the nipple, and glass slides were touched to the secreted drops. The slides were then fixed by immersion in 95\% ethyl alcohol and stained with a Papanicolaou stain. A pathologist with a 10-year experience in the field of breast cytology analysed all cases searching for benign (regular ductal cells, foamy cells, inflammatory cells, red blood cells) or malignant findings (clusters and single-enlarged ductal cells, nuclear pleomorphism, stripped nuclei, nucleoli, necrotic debris). In case of imaging and cytology positivity for papillary lesions or cancer, patients were referred to surgical treatment. In case of negativity, patients were invited to undergo breast MRI in order to exclude any underlying disease.

\section{Imaging technique}

Galactography was performed by accessing the excretory duct with a dedicated 30-gauge cannula (HS, Hospital 
Service, Rome, Italy) and injecting a nonionic iodinated contrast agent (Iopamidol, $300 \mathrm{mg} / \mathrm{mL}$, Bracco Imaging, Milan, Italy) up to a maximum of 1-1.5 mL. After contrast agent administration, each patient underwent FFD mammography and DBT in the craniocaudal, mediolateral oblique, and lateromedial projections during a single breast compression, using the "COMBO" technique (Giotto Tomo, Internazionale Medico Scientifica, Bologna, Italy) consisting of combined acquisition of DBT and FFD mammograms (scanning angle was $40^{\circ}$, step and shoot technique, 13 exposures with reduced optimised dose, pixel size of $86 \mu \mathrm{m}$ ).

\section{Image analysis}

DBT - and FFD-galactography findings were analysed and classified by two independent radiologists with a 10year breast imaging experience. The two readers evaluated FFD-galactograms in a first reading session and DBT images in a second session after 3 weeks, in randomised order, in order to avoid any reading influence. On both DBT and FFD-galactography, all examinations showing filling defects, filling stops or ductal distortion were classified as positive.

\section{Statistical analysis}

Patient population was assessed by summary statistics, and the Kolmogorov-Smirnov test for normal distribution was used in order to test age distribution for normality. Sensitivity, specificity and diagnostic accuracy for FFD and DBT-galactography were calculated having post-surgical histological examination as the reference standard. The obtained performance values were reported as percentages with their 95\% confidence intervals and compared by using the McNemar test. Interobserver agreement was assessed by using Cohen's K. All calculations were performed using the NCSS 2007 software (NCSS, LLC-Kaysville, UT, USA).

\section{Results}

A total of 49 patients (age range, 39-73 years; normal age distribution, $p=0.552$; mean age \pm standard deviation (SD), $50.8 \pm 7.8$ years) with spontaneous, unilateral, single-pore nipple discharge (haematic, $n=29$; serohaematic, $n=20$ ) were enrolled. Forty-three patients $(88 \%)$ were referred to surgical treatment basing on positive imaging findings and nipple discharge cytology. In six cases (12\%), any underlying papillary disease was excluded basing on both imaging findings and cytology.

FFD-galactography was negative in 16/49 (33\%) patients (Fig. 1a) while intraductal lesions were detected in 33/49 (67\%) patients; in particular, 26 (79\%) single intraductal filling defects, 4 (12\%) multiple filling defects (Fig. 2a) and 3 (9\%) filling defects with architectural distortions were found. DBT-galactography was negative in $8 / 49(16 \%)$ patients while intraductal lesions were detected in $41 / 49$ (84\%) patients; in particular, 32 (78\%) single intraductal filling defects (Fig. 1b), 5 (12\%) multiple filling defects (Fig. 2b) and 4 (10\%) filling defects with architectural distortions were found. Nipple discharge cytology was positive in 43/49 (88\%) cases and negative in the remaining 6 . In particular, foamy cells, inflammatory cells, red blood cells, clusters and single enlarged ductal cells were found in 39 cases while clusters and single enlarged ductal cells with nuclear pleomorphism, stripped nuclei, nucleoli and necrotic debris in the remaining 4 . All the negative patients on DBT-galactography $(n=8)$ underwent breast MRI in order to exclude any pathologic condition, and a mass-like enhancement highly suggestive for intraductal papilloma was found in 2 out of 8 patients, with positive cytology in both cases. All patients with pathologic findings $(n=43)$ underwent retroareolar duct excision, and definitive histological examination revealed intraductal disease in all cases: 18/43 (42\%) single intraductal papillomas without atypia, 16/43 (37\%) single intraductal papillomas with atypia, 5/43 (12\%) papillomatosis without atypia and 4/43 (9\%) in situ papillary carcinomas.

Therefore, 41 true positives, 6 true negatives, no false positives and 2 false negatives were found for DBTgalactography; 33 true positives, 6 true negatives, no false positives and 10 false negatives were found for FFD-galactography. Sensitivity, specificity and diagnostic accuracy values of $95 \%, 100 \%$ and $96 \%$ were obtained for DBT-galactography, $77 \%, 100 \%$ and $80 \%$ for FFDgalactography, respectively. In Table 1 , these values are reported together with their 95\% confidence intervals. Statistical analysis (McNemar test) showed a significant difference in favour of DBT for sensitivity $(p=0.008)$ and accuracy $(p=0.038)$, with the same specificity values for both the imaging tools.

An inter-observer agreement of $\kappa=0.86$ was found for DBT-galactography and of $\kappa=0.78$ for FFD-galactography.

\section{Discussion}

Pathologic nipple discharge is typically unilateral, singlepore, spontaneous and persistent, bloody or occurring during postmenopausal phase. According to the patient age, clinical examination with mammography, US and cytological examination of the nipple discharge should be the first line diagnostic approaches. However, no general consensus exists in the medical literature, and cytological examination of nipple discharge has not been found to provide any significant complementary value $[1,6]$. In case of inconclusive mammographic or ultrasonography findings, a second line examination should be considered [17].

Istomin et al. [6] recently reported that galactography is not an obsolete investigation for evaluating pathologic nipple discharge, and it remains a practical, valuable and cost- 


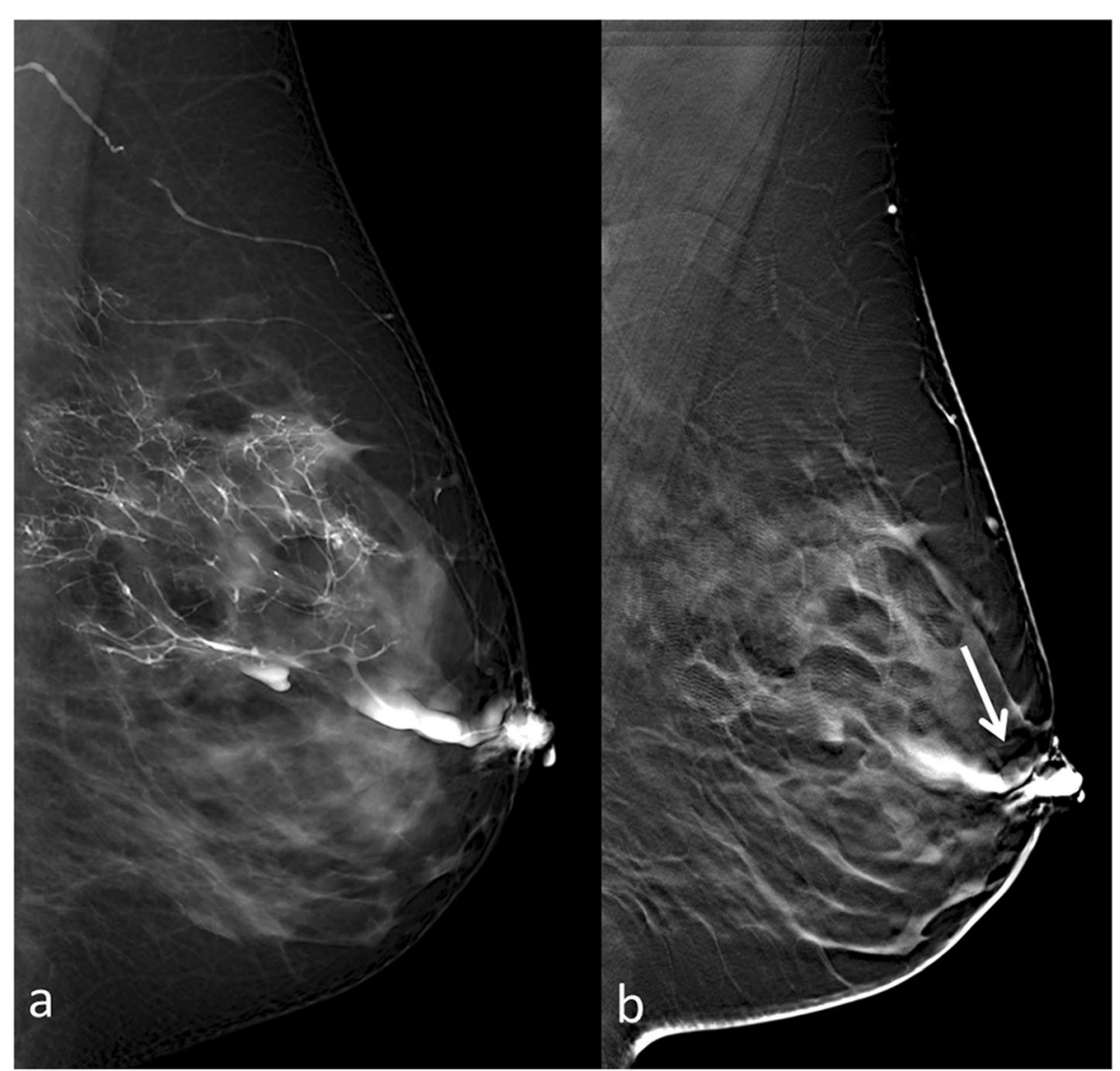

Fig. 1 a Full-field digital (FFD)-galactography. b Digital breast tomosynthesis (DBT)-galactography. Intraductal papilloma of the left breast in a 43year-old patient. The intraductal lesion was not identified on FFD-galactography. Thin section DBT-galactography allows the identification of the intraductal papilloma detectable as a small filling defect (arrow)

effective procedure. Conventional FFD-galactography can still represent a useful diagnostic procedure for evaluating patients with pathologic nipple discharge, since it can provide the location of the underlying lesion when a single duct discharge is identified, in contrast with cytology that can be falsely negative in a high proportion of cases $[6,18]$ and is also unable to provide information on lesion location. Notably, data regarding lesion location is very important for surgical planning. In this field, it has been reported that galactography lesion localisation improves the diagnostic performance of surgical biopsy from the $67 \%$ in non-studied patients to $99 \%$ in patients receiving a galactogram [18].

The galactogram may show normal findings as well as the presence of ductal dilatation, single or multiple filling defects or filling stops. In particular, suspicious findings on galactogram include distortion and ductal wall irregularity highly suggestive of malignancy, even if it is quite challenging to distinguish between malignant and benign lesions only relying on a galactogram [19].

However, FFD-galactography provide only twodimensional images, not always allowing for accurate detection of an intraductal lesion due to overlapping effect and for precise lesion localisation in the threedimensional space. Schulz-Wendtland et al. [16] recently reported a first approach with DBT-galactography ("galactomosynthesis") in only five patients concluding that this kind of imaging tool could be a useful addition to complementary breast diagnostics and could lead to a renaissance of this method. However, their preliminary findings needed to be confirmed in a larger patient population comparing DBT-galactography with FFD-galactography with intra-individual design.

To the best of our knowledge, this is the first study comparing DBT-galactography with FFD-galactography in patients with pathologic nipple discharge. By assessing 49 patients with pathological nipple discharge, we found a significantly higher sensitivity and accuracy of DBTgalactography (95\% and 96\%) than those of FFDgalactography (77\% and $80 \%$, respectively) without any trade-off on terms of sensitivity $(100 \%$ for both techniques). In other words, the gain in accuracy was entirely due to a higher sensitivity.

Our study showed that DBT galactography could represent an accurate tool for identifying and localising 


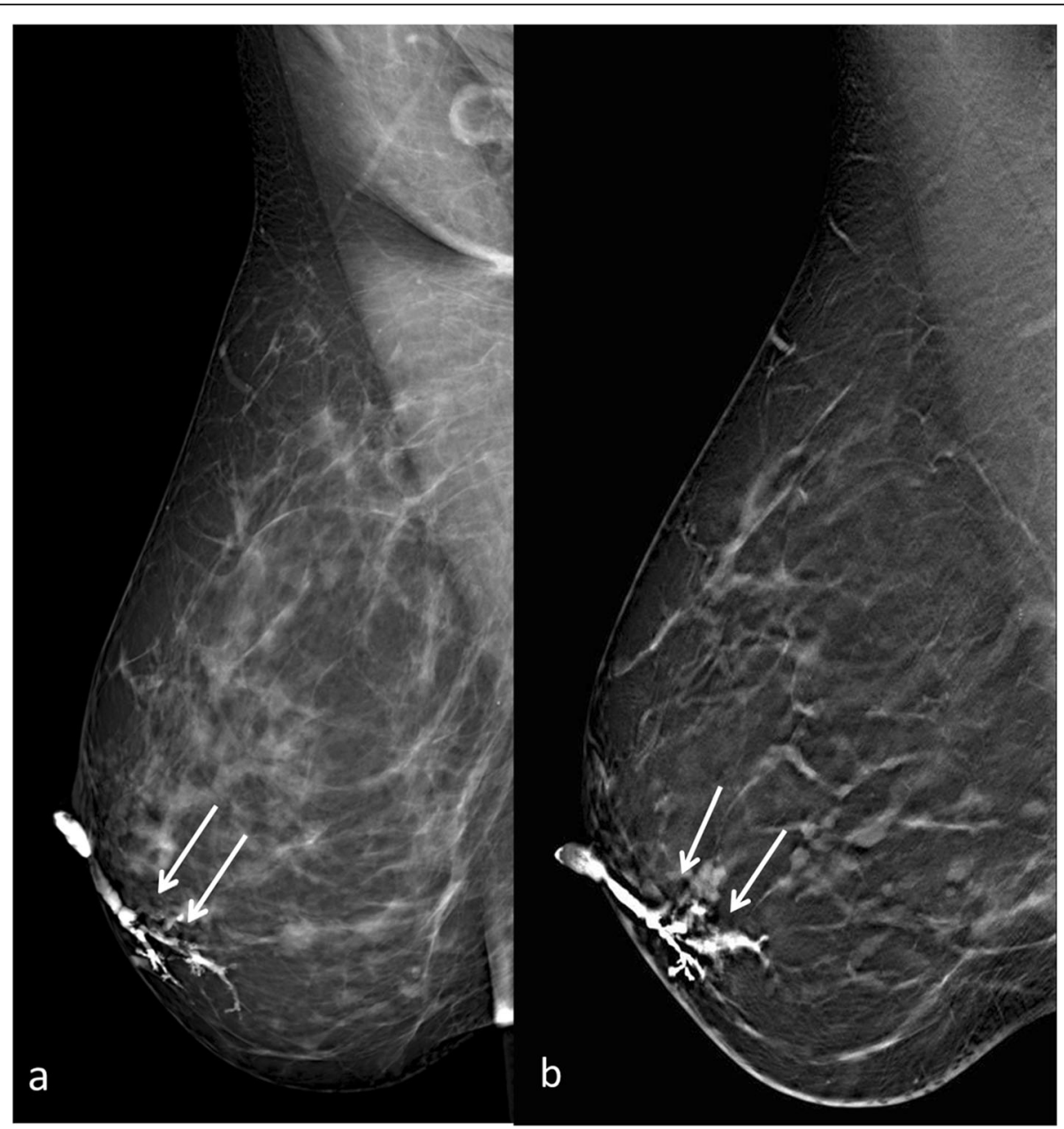

Fig. 2 a Full-field digital (FFD)-galactography. b Digital breast tomosynthesis (DBT)-galactography. Papillomatosis of the right breast in a 42 -yearold patient. The intraductal multiple filling defects are identified on both conventional and DBT-galactography (arrows)

intraductal lesions being the cause of pathologic nipple discharge, notably also characterised by a higher interobserver agreement as compared with FFD-galactography (0.86 versus 0.78 ).

From a general viewpoint, our results confirm the role of DBT in breast imaging, even though further studies of DBT-galactography on larger patient population are still required.

Although DBT-galactography could represent a fast, quite inexpensive, widely available and accurate examination in the field of pathologic nipple discharge, it still remains an invasive procedure to be performed only in case of nipple discharge at the time of the examination and could cause discomfort and pain [1]. On the other side, DBT-galactography uses the same conventional projections of FFD-galactography without significant technical difference. In case of validation on larger series, DBT-galactography could replace FFD-galactography in the workflow of patients with nipple discharge.

Importantly, DBT-galactography could not allow an accurate differential diagnosis between benign or malignant papillary lesions. In this field, MRI provides details

Table 1 Diagnostic performance of digital breast tomosynthesis (DBT)-galactography and full-field digital (FFD)- galactography in 49 patients with pathologic nipple discharge

\begin{tabular}{|c|c|c|c|c|c|c|c|c|c|c|}
\hline & \multirow[t]{2}{*}{ TP } & \multirow[t]{2}{*}{$\mathrm{TN}$} & \multirow[t]{2}{*}{ FP } & \multirow[t]{2}{*}{ FN } & \multicolumn{2}{|l|}{ Sensitivity (\%) } & \multicolumn{2}{|l|}{ Specificity (\%) } & \multicolumn{2}{|l|}{ Accuracy (\%) } \\
\hline & & & & & Point estimate & $95 \% \mathrm{Cl}$ & Point estimate & $95 \% \mathrm{Cl}$ & Point estimate & $95 \% \mathrm{Cl}$ \\
\hline DBT-galactography & 41 & 6 & 0 & 2 & 95.3 & $84.2-99.4$ & 100.0 & $54.1-100.0$ & 95.9 & $86.0-99.5$ \\
\hline FFD-galactography & 33 & 6 & 0 & 10 & 76.7 & $61.4-88.2$ & 100.0 & $54.1-100.0$ & 79.6 & $65.7-89.8$ \\
\hline
\end{tabular}

Cl Confidence interval, FN False negatives, FP False negatives, TN True negatives, TP True positives 
in addition to the morphological information of DBTgalactography with a higher potential for lesion characterisation basing on the enhancement features $[1,10$, 20]. In fact, MRI allows an accurate imaging of both breasts detecting multifocal, multicentre or occult lesions evaluating at the same time all the ductal system also in the deepest areas of the breast as compared with galactography which allows to study only segmental ductal areas. MRI has also been reported as an accurate tool for the management of nipple discharge especially in case of negative or inconclusive mammography or for a correct preoperative evaluation; despite the higher costs, it allows to exclude malignant lesions with high accuracy and to avoid unnecessary surgical procedures with a crucial role for clinical management $[20,21]$. Of note, in our study, MRI has been considered as the next step in case of patients with negative findings on both FFD- and DBT-galactography. However, further studies comparing unenhanced plus contrast-enhanced MRI with DBT-galactography are needed. Finally, the recent introduction of contrast-enhanced mammography [10] needs to be investigated also in patients with pathological nipple discharge to allow for diagnosing benign, borderline or malignant lesions associated with this clinical condition.

We have also to consider that contraindications do exist for DBT-galactography mainly represented by severe allergy to iodinated contrast material, nipple retraction or a history of a prior nipple surgery that would invalidate the examination, as is for conventional galactography [1].

Our study has limitations. First, we did not evaluated differences in terms of average glandular dose (AGD) between the two techniques. This was due to the fact that we used the COMBO mode in which the two imaging techniques are acquired in a combined fashion that prevented a separation of the two doses. However, we can consider that entire AGD was $1.94 \pm 0.64$. This AGD, even all attributed to the DBT, means that it still remains acceptable for the clinical use, and in case of validation on larger series, the use of DBT-galactography with synthetically reconstructed images will certainly reduce the previous AVG value. Second, we studied a relatively small number of patients that, however, allowed to show a significant difference in terms of sensitivity and accuracy in favour of DBT, but not to get information about possible false positive cases that we did not encounter in our case series for both techniques.

In conclusion, we showed that DBT-galactography provided a sensitivity and a diagnostic accuracy higher than that of FFD-galactography. It could represent a reliable and largely available diagnostic tool in patients with pathologic nipple discharge, potentially avoiding the need for MRI in this clinical setting.

\section{Abbreviations}

AGD: Average glandular dose; DBT: Digital breast tomosynthesis; FFD: Fullfield digital; MRI: Magnetic resonance imaging

\section{Authors' contributions}

MM, VDR, and MT conceived and designed the study. MM, VDR, and MT acquired the data. MM, VDR, AD, NT, SP, and MT analysed and interpreted the data. MM, AD, and MT drafted the manuscript. All authors revised the manuscript. All authors read and approved the final manuscript.

\section{Funding}

This research was financially supported by The Susan G. Komen Italy - Breast Cancer Foundation - 2018 Breast Cancer Research Project.

\section{Availability of data and materials}

The relevant data have been included in the manuscript. The datasets used and/or analysed during the current study are available from the corresponding author on reasonable request.

Ethics approval and consent to participate

Institutional review board approval and patients' informed consent were obtained.

\section{Consent for publication}

Consent to publish was obtained from patients.

\section{Competing interests}

All other authors declare that they have no competing interests.

\section{Author details}

'DETO-Department of Emergency and Organ Transplantation-Breast Care Unit, Aldo Moro University of Bari Medical School, Piazza Giulio Cesare 11, 70124 Bari, Italy. ${ }^{2}$ DIM-Interdisciplinary Department of Medicine-Section of Diagnostic Imaging, Aldo Moro University of Bari Medical School, Piazza Giulio Cesare 11, 70124 Bari, Italy.

Received: 1 February 2020 Accepted: 17 June 2020

Published online: 04 August 2020

\section{References}

1. Panzironi G, Pediconi F, Sardanelli F (2018) Nipple discharge: the state of art. BJR Open 1:20180016. https://doi.org/10.1259/bjro.20180016

2. Chen L, Zhou WB, Zhao Y et al (2012) Bloody nipple discharge is a predictor of breast cancer risk: a meta-analysis. Breast Cancer Res Treat 132:9-14. https://doi.org/10.1007/s10549-011-1787-5

3. Wei S (2016) Papillary lesions of the breast: an update. Arch Pathol Lab Med 140:628-643. https://doi.org/10.5858/arpa.2015-0092-RA

4. Sauter ER, Schlatter L, Lininger J, Hewett JE (2004) The association of bloody nipple discharge with breast pathology. Surgery 136:780-785. https://doi. org/10.1016/j.surg.2004.06.017

5. Rageth CJ, O'Flynn EA, Comstock C et al (2016) First International Consensus Conference on lesions of uncertain malignant potential in the breast (B3lesions). Breast Cancer Res Treat 159:203-213. https://doi.org/10.1007/ s10549-016-3935-4

6. Istomin A, Masarwah A, Pitkänen M et al (2018) Galactography is not an obsolete investigation in the evaluation of pathological nipple discharge. PLoS One 13:e0204326. https://doi.org/10.1371/journal.pone.0204326

7. Orel SG, Dougherty CS, Reynolds C, Czerniecki BJ, Siegelman ES, Schnall MD (2000) MR imaging in patients with nipple discharge: initial experience. Radiology 216:248-254. https://doi.org/10.1148/radiology.216.1.r00jn28248

8. Francis A, England D, Rowlands D, Bradley S (2002) Breast papilloma: mammogram, ultrasound and MRI appearances. Breast 11:394-397. https:// doi.org/10.1054/brst.2002.0452

9. Ballesio L, Maggi C, Savelli S et al (2007) Adjunctive diagnostic value of ultrasonography evaluation in patients with suspected ductal breast disease. Radiol Med 112:354-365. https://doi.org/10.1007/s11547-007-0146-4

10. Berger N, Luparia A, Di Leo G et al (2017) Diagnostic performance of MRI versus galactography in women with pathologic nipple discharge: a systematic review and meta-analysis. AJR Am J Roentgenol 209:465-471. https://doi.org/10.2214/AJR.16.16682 
11. Funovics MA, Philipp MO, Lackner B, Fuchsjaeger M, Funovics PT, Metz V (2003) Galactography: method of choice in pathologic nipple discharge? Eur Radiol 13:94-99. https://doi.org/10.1007/s00330-002-1401-7

12. Cardenosa G, Doudna C, Eklund GW (1994) Ductography of the breast: technique and findings. AJR Am J Roentgenol 162:1081-1087. https://doi. org/10.2214/ajr.162.5.8165986

13. Hou MF, Huang TJ, Liu GC (2001) The diagnostic value of galactography in patients with nipple discharge. Clin Imaging 25:75-81. https://doi.org/10 1016/s0899-7071(01)00256-x

14. Dinkel HP, Trusen A, Gassel AM, Rominger M et al (2000) Predictive value of galactographic patterns for benign and malignant neoplasms of the breast in patients with nipple discharge. Br J Radiol 73:706-714. https://doi.org/10. 1259/bjr.73.871.11089460

15. Bahl M, Mercaldo S, Vijapura CA, McCarthy AM, Lehman CD (2019) Comparison of performance metrics with digital $2 \mathrm{D}$ versus tomosynthesis mammography in the diagnostic setting. Eur Radiol 29:477-484. https://doi. org/10.1007/s00330-018-5596-7

16. Schulz-Wendtland R, Preuss C, Fasching PA et al (2018) Galactography with tomosynthesis technique (galactomosynthesis) - renaissance of a method? Geburtshilfe Frauenheilkd 78:493-498. https://doi.org/10.1055/a-0594-2277

17. Sheiman LS, Levesque PH (2016) The in's and out's of ductography: a comprehensive review. Curr Probl Diagn Radiol 45:61-70. https://doi.org/10. 1067/j.cpradiol.2015.05.007

18. Zervoudis S, latrakis G, Economides P, Polyzos D, Navrozoglou I (2010) Nipple discharge screening. Womens Health (Lond) 6:135-151. https://doi. org/10.2217/whe.09.81

19. Lee SJ, Trikha S, Moy L et al (2017) ACR Appropriateness Criteria ${ }^{\circledR}$ evaluation of nipple discharge. J Am Coll Radiol 14:S138-S153. https://doi.org/10.1016/ j.jacr.2017.01.030

20. Bahl M, Gadd MA, Lehman CD (2017) Journal club: diagnostic utility of MRI after negative or inconclusive mammography for the evaluation of pathologic nipple discharge. AJR Am J Roentgenol 209:1404-1410. https:// doi.org/10.2214/AJR.17.18139

21. Zanardo M, Cozzi A, Trimboli RM et al (2019) Technique, protocols and adverse reactions for contrast-enhanced spectral mammography (CESM): a systematic review. Insights Imaging 10:76. https://doi.org/10.1186/s13244019-0756-0

\section{Publisher's Note}

Springer Nature remains neutral with regard to jurisdictional claims in published maps and institutional affiliations.

\section{Submit your manuscript to a SpringerOpen ${ }^{\circ}$ journal and benefit from:}

- Convenient online submission

- Rigorous peer review

- Open access: articles freely available online

- High visibility within the field

- Retaining the copyright to your article

Submit your next manuscript at $\boldsymbol{\nabla}$ springeropen.com 